\title{
Disgust- and not fear-evoking images hold our attention
}

\author{
Johanna C. van Hooff ${ }^{\text {a,* }}$, Christel Devue ${ }^{\text {a,b }}$, Paula E. Vieweg ${ }^{\text {c }}$, Jan Theeuwes ${ }^{\text {a }}$ \\ a Cognitive Psychology Department, VU University Amsterdam, Amsterdam, The Netherlands \\ b Cognitive Science Department, Université de Liège, Liège, Belgium \\ c University of Amsterdam, Amsterdam, The Netherlands
}

\section{A R T I C L E I N F O}

\section{Article history:}

Received 25 September 2012

Received in revised form 28 January 2013

Accepted 1 February 2013

Available online xxxx

\section{PsycINFO classification:}

2346 (Attention)

2360 (Motivation \& Emotion)

\section{Keywords:}

Emotion

Disgust

Fear

Threat

Attention bias

Disengagement

\begin{abstract}
A B S T R A C T
Even though disgust and fear are both negative emotions, they are characterized by different physiology and action tendencies. The aim of this study was to examine whether fear- and disgust-evoking images would produce different attention bias effects, specifically those related to attention (dis)engagement. Participants were asked to identify a target which was briefly presented around a central image cue, which could either be disgusting, frightening, or neutral. The interval between cue onset and target presentation varied within blocks $(200,500,800,1100 \mathrm{~ms})$, allowing us to investigate the time course of attention engagement. Accuracy was lower and reaction times were longer when targets quickly (200 ms) followed disgust-evoking images than when they followed neutral- or fear-evoking images. For the other, longer interval conditions no significant image effects were found. These results suggest that emotion-specific attention effects can be found at very early visual processing stages and that only disgust-evoking images, and not fear-evoking ones, keep hold of our attention for longer. We speculate that this increase in early attention allocation is related to the need to perform a more comprehensive risk-assessment of the disgust-evoking images. The outcomes underline not only the importance of examining the time course of emotion induced attention effects but also the need to look beyond the dimensions of valence and arousal.
\end{abstract}

(c) 2013 Elsevier B.V. All rights reserved.

\section{Introduction}

Through selective attention, our brain guides our behavior toward environmental stimuli that are relevant for survival, especially when they imply immediate danger (Vuilleumier, 2005). Evidence for this notion, comes from experimental and clinical studies that have used a variety of stimuli (e.g., pictures, faces, words) and paradigms that tap into different aspects of attention (for reviews see, Bar-Haim, Lamy, Pergamin, Bakermans-Kranenburg, \& Van IJzendoorn, 2007; Cisler, Bacon, \& Williams, 2009; Weierich, Treat, \& Hollingworth, 2008; Yiend, 2010). Results from most of these studies suggest that there exists an attention bias toward threatening or fear-evoking stimuli, particularly in high-anxious individuals. Results however, do not conclusively indicate whether this attention bias constitutes facilitated attention toward threatening stimuli, impaired disengagement from them, or both (e.g., Fox, Russo, Bowles, \& Dutton, 2001; Koster, Crombez, Van Damme, Verschuere, \& De Houwer, 2004; Koster, Crombez, Verschuere, Van Damme, \& Wiersema, 2006). Methodological variables, such as stimulus duration and stimulus threat value, as well as individual differences in anxiety seem to modulate the

\footnotetext{
* Corresponding author at: Department of Cognitive Psychology, VU University Amsterdam, Van der Boechorststraat 1, 1081 BT Amsterdam, The Netherlands. Tel.: +31 20 5985577; fax: + 31205988971.

E-mail address: J.C.van.Hooff@vu.nl (J.C. van Hooff).
}

pattern of results (Cisler, Olatunji, Lohr, \& Williams, 2009; Mogg \& Bradley, 1998).

One remarkable observation in many of these studies is that researchers often refer to effects of 'threatening' or 'fear-evoking' information, while they have actually used stimulus materials that are 'negative' and 'arousing', according to the database they were selected from (e.g., Affective Norms for English Words - ANEW, Bradley \& Lang, 1999; International Affective Picture System - IAPS, Lang, Bradley, \& Cuthbert, 2008). While threatening words and pictures are evidently negative and most times arousing, not all negative stimuli are necessarily threatening or fear-evoking. Moreover, a large number of negative, arousing IAPS images that are typically classified as highly threatening (e.g., pictures showing injuries, mutilations, or burn victims) have in fact been found to elicit stronger feelings of 'disgust' than of 'fear' (Libkuman, Otani, Kern, Viger, \& Novak, 2007; Mikels et al., 2005). It is therefore disputable whether the previously reported attention bias effects (particularly those with reference to IAPS pictures) can be attributed solely to fear and the purely threatening nature of the stimuli used. Indeed, in such cases, it is more appropriate to explain the observed effects in terms of the stimulus selection criteria applied, namely valence (e.g., Pratto \& John, 1991) or arousal (e.g., Schimmack, 2005; Vogt, De Houwer, Koster, Van Damme, \& Crombez, 2008). Nevertheless, this does not rule out the possibility that there are emotion-specific attention effects, which may be unique for fear- or disgust-evoking images. 
Given that the emotions fear and disgust activate different brain areas (Calder, Lawrence, \& Young, 2001; Murphy, Nimmo-Smith, \& Lawrence, 2003) and are linked to different action tendencies (Susskind et al., 2008), it is feasible that they also affect attention differentially. Moreover, while fear is believed to enhance sensory acquisition in order to deal quickly and efficiently with threatening circumstances, disgust more likely serves the function of diminishing environmental input in order to avoid contamination (Susskind et al., 2008). Furthermore, while the first process is believed to be fast and largely automatic, the latter presumably develops more slowly and depends more on focal attention (Anderson, Christoff, Panitz, De Rosa, \& Gabrieli, 2003; Santos, Iglesias, Olivares, \& Young, 2008). Consequently, if fear- and disgust-evoking stimuli indeed affect attention differently then it seems unjustified to treat them as one single category. Moreover, an inconsistent use of negative, arousing stimulus materials that could be either, threatening, disgusting, or both, could have been responsible for some of the contrasting findings reported in the literature.

Hardly any behavioral studies have directly compared attention effects for threat-related versus disgust-related stimuli, but those that have done so found larger attention biases for the latter. For example, using a modified Stroop task, Charash and McKay (2002) found that color-naming of disgust-related words (e.g., vomit) was delayed relative to neutral words (e.g., igloo), while a similar effect was not present for fear-related words (e.g., tumor). Using the same sets of words in a rapid serial visual processing paradigm (RSVP), Cisler, Olatunji, et al. (2009) found that probes were more difficult to detect following disgust targets than following fear targets. Results from both experiments suggest that attention disengagement is more difficult from disgust-related words than from fear-related words. In a recent event-related potential (ERP) study, similar results were found for pictorial stimuli (Carretié, Ruiz-Padial, López-Martín, \& Albert, 2011). Participants in this study responded slower and less accurate in a digit categorization task when the targets were presented superimposed on disgust pictures than on fearful or neutral pictures. In addition, only the disgust pictures were found to elicit larger P2 components than the neutral pictures, suggesting that specifically these images, and not the fearful ones, received more attention during early perceptual processing. In contrast, Ciesielski, Armstrong, Zald, and Olatunji (2010) observed an equal drop in detection accuracy when targets were presented in a RSVP stream following fear- and disgust-image distracters as compared to neutral ones. In addition, for both types of negative images detection accuracy became gradually better with longer time lags, suggesting a similar development in attention allocation over time. Nevertheless, close inspection of their data also showed that at the shortest time lag (200 ms), accuracy was slightly lower following disgust- as compared to fear-images, suggesting somewhat greater attention allocation to the disgust pictures at early processing stages.

The main aim of the present study was to provide further evidence that fear- and disgust-evoking images produce different attention bias effects, perhaps not only in magnitude but also in onset and duration. First, we carefully selected our stimulus materials by means of an independent rating study, ensuring that the pictures (from the IAPS database) generated feelings of fear or disgust, while keeping their arousal and valence ratings constant. We then employed a covert orienting paradigm (modeled after Fox et al., 2001, Experiment 5) to investigate whether these two sets of negative pictures would hold participants' attention to different extent and/or at different times following picture onset. In Fox et al.'s paradigm, task-irrelevant affective cues were presented at fixation, in focus of attention, while participants were required to identify a target that was briefly presented in the surrounding of this cue after a brief interval. Difficulty with disengaging attention from the central image cue would result in prolonged target identification times. Indeed, while using a cue-target interval of $600 \mathrm{~ms}$, Fox et al. (2001) found that anxious individuals took longer to identify a peripheral target when they fixated on a threat-related word than when they fixated on a neutral or positive word.

In a novel adaptation of this paradigm, we systematically varied the time interval between cue onset and target presentation, which allowed us to investigate not only the existence and magnitude of attention (dis)engagement but also its time course. In general, the longer the cue-target interval, the more time participants have to disengage their attention from the central image cue. At relatively longer cue-target intervals, it can therefore be expected that response delays for the distinct emotion cues are no longer present or even show the opposite pattern, in case of attention avoidance (cf., Mogg, Bradley, Miles, \& Dixon, 2004). In the current experiment, we used cue-target intervals of $200,500,800$, and $1100 \mathrm{~ms}$, respectively. These intervals were chosen to enable investigation of emotion-specific differences (fear versus disgust) in both early attention (dis)engagement and subsequent attention avoidance.

Based on previous research, we hypothesized that it would take longer to identify targets paired with negative image cues (both disgust- and fear-evoking) than those paired with neutral cues (e.g., Fox et al., 2001; Koster et al., 2006; Vogt et al., 2008). Furthermore, in accord with the findings by Carretié et al. (2011), such response delay was expected to be more prominent for the disgust-evoking images than for the fear-evoking images, reflecting superior attention holding capacities of the former. Finally, given the respective functions of fear and disgust (e.g., Susskind et al., 2008), we reasoned that attention engagement effects for fear-evoking images may be more transient to those of the disgust-evoking images (thus response delays more restricted to the shorter cue-target intervals) and less likely to convert into attention avoidance (thus no quicker target identification at the longest cue-target interval).

\section{Method}

\subsection{Participants}

Thirty female university students took part in this experiment, earning either course credits or $5 €$ for their participation. They were aged between 19 and 30 years ( $M=21.0$ years). Only females were recruited because our stimulus selection procedure (see below) revealed significant gender differences as to how the IAPS pictures were rated in terms of arousal and valence (see also, Lang et al., 2008; Weinberg \& Hajcak, 2010). In addition, previous studies have shown that women are characteristically more disgust sensitive than men (Charash, McKay, \& DiPaolo, 2006) and display greater vigilance for threat-related information (Dickie \& Armony, 2008). Using an all-female sample would therefore remove a confounding variable.

\subsection{Stimulus selection}

Forty IAPS pictures were selected for the experimental task on the basis of an independent rating study consisting of 160 IAPS pictures (Lang et al., 2008). For this rating study, 40 pictures were chosen based on their estimated likelihood of generating feelings of disgust. These pictures depicted dead animals, dirty toilets, contaminated food, crawling animals (e.g., cockroaches, maggots), and disgusting actions (e.g. vomiting). Forty other pictures were chosen based on their likelihood of eliciting feelings of fear. They depicted aggressive animals (e.g., dogs, sharks), pointed guns, violent actions, and dangerous scenes (e.g., riots, car accidents). Pictures that were believed to generate feelings of both fear and disgust (e.g., mutilation, spiders) were discarded. Finally, eighty neutral pictures were chosen, showing tranquil animals, household objects, peaceful scenes, and simple everyday actions (e.g., typing, reading). All pictures were adjusted into equally sized squares.

Twenty-seven independent judges (15 females, 18-24 years) were asked to rate arousal and valence of the 160 chosen IAPS pictures 
Table 1

Mean rating scores (standard deviations in parentheses) for the 40 selected IAPS pictures based on the independent stimulus selection rating study.

\begin{tabular}{|c|c|c|c|c|}
\hline & $\begin{array}{l}\text { Neutral pictures } \\
(n=20)\end{array}$ & $\begin{array}{l}\text { Fear pictures } \\
(\mathrm{n}=10)\end{array}$ & $\begin{array}{l}\text { Disgust pictures } \\
(\mathrm{n}=10)\end{array}$ & $\begin{array}{l}\text { t-test results } \\
\text { (fear vs disgust) }\end{array}$ \\
\hline Arousal rating & $1.86(0.31)$ & $5.40(0.42)$ & $5.64(0.54)$ & $\mathrm{t}(18)=1.09 ; p=.29$ \\
\hline Valence rating & $6.54(0.46)$ & $2.76(0.41)$ & $2.52(0.43)$ & $\mathrm{t}(18)=1.28 ; p=.22$ \\
\hline Fear rating & $1.00(0.00)$ & $3.24(0.32)$ & $1.57(0.24)$ & $\mathrm{t}(18)=13.33 ; p<.001$ \\
\hline Disgust rating & $1.00(0.00)$ & $2.08(0.35)$ & $4.06(0.33)$ & $\mathrm{t}(18)=13.02 ; p<.001$ \\
\hline
\end{tabular}

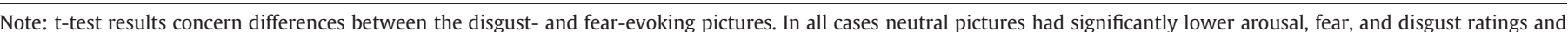

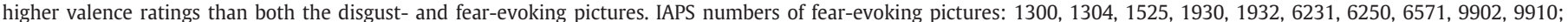

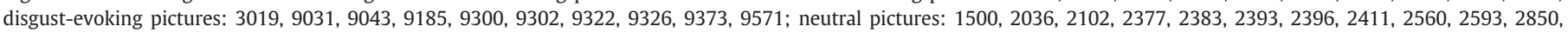
5390, 7001, 7026, 7041, 7050, 7057, 7235, 7493, 7513.

by means of a 9-point rating scale using the Self-Assessment Manikin (SAM; Bradley \& Lang, 1994). Feelings of disgust and fear were subsequently assessed by two separate 5-point Likert scales that varied from "not at all" (1), via "somewhat" (3), to "very much" (5). Participants were asked to respond promptly and according to their first impression.

Stimulus selection was based on the averaged responses of the 15 female participants, because arousal ratings from our male participants turned out to be generally low and not sufficiently different for the three stimulus categories (fear, disgust, neutral). The following criteria were used to select the 10 threat- and 10 disgust-related images for the experimental task: (1) images should have the highest and lowest fear-minus-disgust absolute difference scores to ensure optimal separation between the two negative emotions, (2) disgusting and threatening images should be within the top 30\% of individual disgust or fear scores, respectively, and (3) there should be no significant differences in averaged arousal and valence ratings between the selected fear- and disgust-evoking pictures. Twenty neutral pictures, with minimal fear and disgust ratings, were matched as best as possible with the negative pictures on the basis of their visual complexity and figure-background composition. Almost all selected pictures depicted only one object, one animal, or one person performing a simple act. ${ }^{1}$ The neutral pictures had significantly higher valence ratings and significantly lower arousal ratings than the negative pictures. There were twice as many neutral images to equate the number of emotion versus neutral pictures and to prevent participants from developing a negative mood. Characteristics of the 40 selected pictures are indicated in Table 1.

\subsection{Procedure}

Before the experiment, participants were asked to fill in Dutch versions of the State-Trait Anxiety Inventory (STAI; Ploeg, Defares, \& Spielberger, 1980) and the Revised Disgust Scale (DS-R; Olatunji et al., 2007). Then, the covert orienting task was explained followed by two training blocks of 24 trials each.

Fig. 1 describes the sequence of events in one example trial of the task. Each trial began with the presentation of a central fixation cross (1000 ms), which was followed by an image cue $\left(231 \times 231\right.$ pixels; $7^{\circ}$ visual angle viewed at $75 \mathrm{~cm}$ ) presented in the middle of the screen. Shortly after cue onset, a target (letter $\mathrm{Z}$ or N, font Arial, size 7.5) appeared for $50 \mathrm{~ms}$ either above, below, left, or right of the image cue (approximately $4.5^{\circ}$ visual angle from the center of the screen). The cue-target interval varied randomly within test blocks and could be either $200 \mathrm{~ms}, 500 \mathrm{~ms}, 800 \mathrm{~ms}$, or $1100 \mathrm{~ms}$. There were 16 test blocks, each composed of 40 trials (10 disgust, 10 fear, 20 neutral) and interrupted by breaks. Each picture was paired with each cue-target interval and each of these combinations was repeated

\footnotetext{
${ }^{1}$ For the fear-evoking category there were two exceptions to this rule, one image depicted a gun attack on another person (matched in the neutral category with two people in discussion on a terrace), and one image depicted rescue workers around a car wreckage (matched in the neutral category with several people having a picnic).
}

four times, giving a total of 640 trials. The identity and the position of the target (above, below, left, right) varied at random. Each test block could be started by means of a button press by the participants. Their task was to indicate as accurately and quickly as possible which target was presented ( $\mathrm{Z}$ or $\mathrm{N}$ ) by pressing the corresponding keyboard button, with their left or right index finger, respectively. The image cue remained on the screen until a response was made with a maximum of $1200 \mathrm{~ms}$. Image cue offset was followed by a $500 \mathrm{~ms}$ blank screen or a feedback screen if no response was made. Participants had to remain fixated on the center of the screen. As the presentation time of the target letter was only $50 \mathrm{~ms}$, it was impossible to make a directed eye movement to the target letter.

\section{Results}

Mean questionnaire scores were STAI-state $=34.1$ (SD 7.7, range 22-57), STAI-trait $=37.0$ (SD 8.2, range 22-60), and DS-R $=53$ (SD 7.6, range 30-86). STAI-state and STAI-trait scores correlated with each other $(r=0.79, p<0.001)$, but both did not correlate with DS-R scores. Two participants obtained relatively high STAI - state/trait anxiety scores (one participant $57 / 54$ and the other $51 / 60$ respectively). Two other participants obtained relatively high disgust-sensitivity scores (total scores of 79 and 86 respectively). Analyses with or without these four participants produced highly similar results; those in which they are included are reported.

Accuracy and RT were each analysed with a 4 (Interval: 200, 500, $800,1100 \mathrm{~ms}) \times 3$ (Stimulus: fear, disgust, neutral) repeated measures ANOVA. Accuracy ranged from 0.69 to 0.97 , with an average of 0.85 correct target identifications. A significant main effect of interval was present $\left(F(3,87)=5.39, p<0.01, \eta_{p}{ }^{2}=0.16\right)$ as well as a significant Interval $\times$ Stimulus interaction $\left(F(6,174)=2.57, p<0.05, \eta_{p}{ }^{2}=\right.$ 0.08 ). Average accuracy was generally lower in the $200 \mathrm{~ms}$ interval condition $\left(M_{200}=0.83\right)$ as compared to the other intervals $\left(M_{500}=\right.$ $\left.0.86, M_{800}=0.87, M_{1100}=0.86\right)$. Follow-up analyses per cue-target interval showed that the effect of stimulus was significant for the $200 \mathrm{~ms}$ interval condition only $\left(\mathrm{F}(2,58)=5.24, p<0.05, \eta_{p}{ }^{2}=0.15\right)$ with less accurate target identifications for the disgust-evoking pictures $\left(M_{\text {disgust }}=0.80\right)$ as compared to the neutral pictures $\left(M_{\text {neutral }}=0.85\right.$, $p<0.05)$ and the fear-evoking pictures $\left(M_{\text {fear }}=0.85, p=0.07\right)$.

Mean RT values for each cell are presented in Fig. 2, calculated for correct responses only. RTs shorter than $200 \mathrm{~ms}$ were considered guesses and were excluded from analyses. Significant main effects were found for both interval $\left(F(3,87)=91,9, p<0.001, \eta_{p}^{2}=0.76\right)$ and stimulus $\left(F(2,58)=5.94, p<0.01, \eta_{\mathrm{p}}^{2}=0.17\right)$ as well as a significant Stimulus $\times$ Interval interaction $\left(F(6,174)=9.33, p<0.001, \eta_{p}^{2}=\right.$ $0.24)$. With regards to the main effect of interval, RTs for the shortest cue-target interval $\left(M_{200}=550 \mathrm{~ms}\right)$ were slower compared to all other intervals $\left(M_{500}=501 \mathrm{~ms} ; M_{800}=497 ; M_{1100}=513 \mathrm{~ms}\right.$; all $p s<0.001$ ). With regards to the main effect of stimulus, only one pairwise comparison reached significance, revealing that, across the different cue-target intervals, RTs were slower when targets were paired with a disgust-evoking picture $\left(M_{\text {disgust }}=520 \mathrm{~ms}\right)$ as compared to a neutral picture $\left(M_{\text {neutral }}=512 \mathrm{~ms}\right)(p<0.01)$. Then again, 


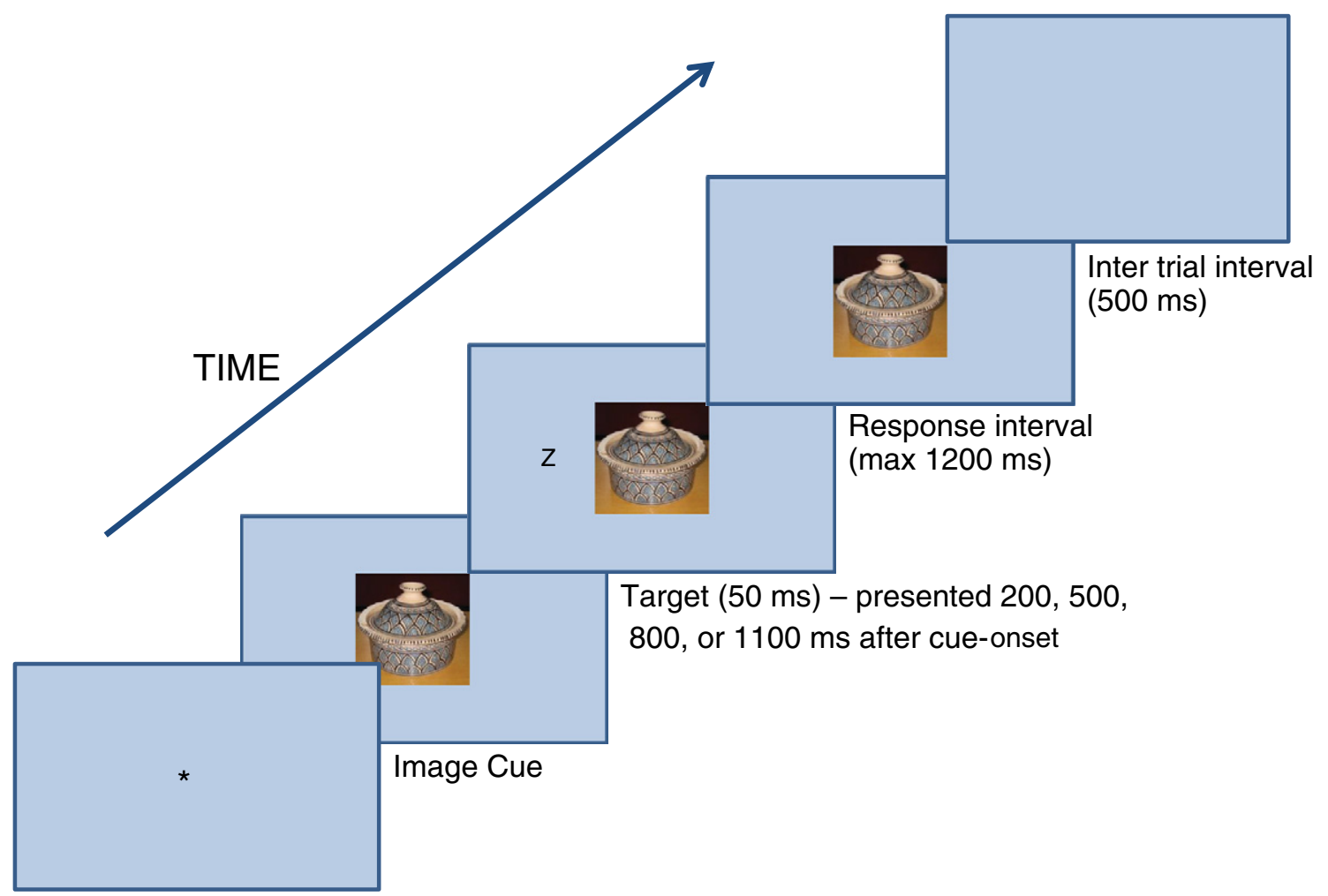

Fixation (1000 ms)

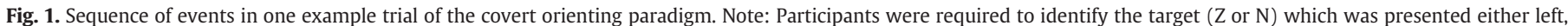

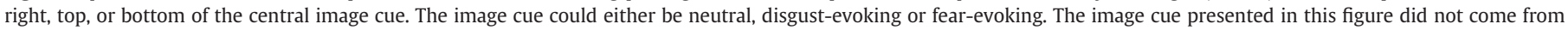
the IAPS database but is given as an example for the neutral condition.

as confirmed by the significant interaction, the stimulus effects were almost exclusively due to the RT differences observed for the $200 \mathrm{~ms}$ interval condition (see also Fig. 2). Moreover, follow-up ANOVAs for each cue-target interval revealed that the stimulus effect was significant for the $200 \mathrm{~ms}$ interval condition only $(F(2,58)=25.6, p<0.001$, $\eta_{\mathrm{p}}^{2}=0.47$ ), with longer target identification times for the disgustevoking pictures $\left(\mathrm{M}_{\text {disgus__200 }}=571 \mathrm{~ms}\right)$ as compared to both the neutral $\left(\mathrm{M}_{\text {neutral_200 }}=537 ; p<0.001\right)$ and fear-evoking pictures $\left(\mathrm{M}_{\text {fear_200 }}=\right.$ $541 ;(p<0.001)$. This delay in RT did not correlate with any of the individual difference variables (i.e., disgust sensitivity, trait anxiety, state anxiety).

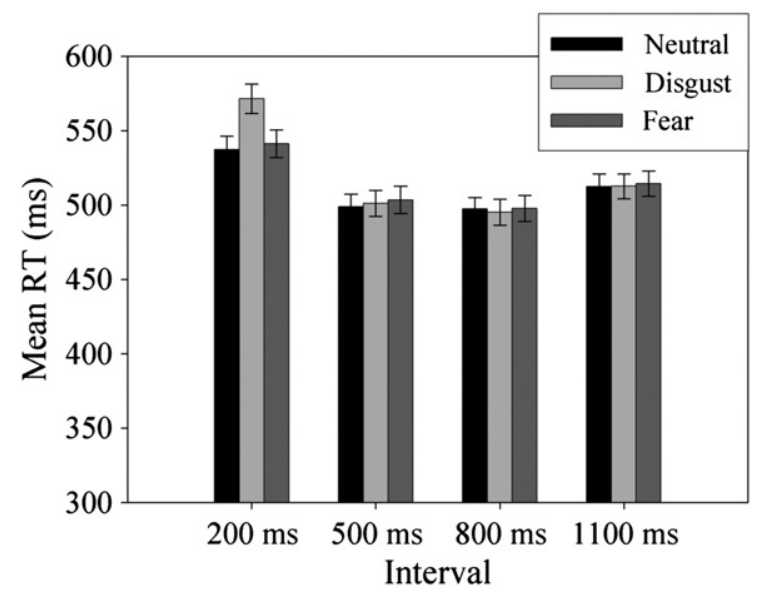

Fig. 2. Mean reaction time results for each stimulus type (neutral, disgust, fear) as a function of cue-target interval $(200,500,800,1100 \mathrm{~ms})$.
To examine the possibility that accuracy and RT results for the $200 \mathrm{~ms}$ cue-target interval condition were caused by different habituation rates for the two sets of negative stimuli, we divided the data set for this shortest interval into four equal-sized segments and carried out a 4 (Test block: $1-4,5-8,9-12,13-16) \times 3$ (Stimulus: fear, disgust, neutral) repeated measures ANOVA. For accuracy, no significant effects of test block nor interactions with this factor were found. For RT however, a significant main effect of test block was present $\left(F(3,87)=24.5, p<0.001, \eta_{p}{ }^{2}=0.46\right)$ as well as a significant Test block $\times$ Stimulus interaction $\left(F(6,174)=4.47, p<0.001, \eta_{p}{ }^{2}=0.13\right)$. RTs were slower for the first test blocks $\left(M_{\text {block1-4 }}=591 \mathrm{~ms}\right)$ as compared to all other test blocks $\left(M_{\text {block5-8 }}=552 \mathrm{~ms}, M_{\text {block9-12 }}=531 \mathrm{~ms}\right.$, $M_{\text {block13-16 }}=524 \mathrm{~ms}$ ) (all ps $\left.<0.01\right)$. RTs for blocks 5-8 were also significantly slower compared to those for blocks 9-12 and blocks 13-16 (both $p s<0.01$ ). General response speed thus became gradually faster over the course of the experiment with no significant drop in accuracy. More interestingly, stimulus effects on RT also became gradually smaller, with the largest effects in blocks $1-4(F(2,58)=16.1$, $p<0.001)$, somewhat smaller effects in blocks $5-8(\mathrm{~F}(2,58)=5.38$, $p<0.01)$ and blocks $9-12(\mathrm{~F}(2,58)=5.58, p<0.01)$, and no significant effects in blocks 13-16. Similar to the overall results discussed above, these stimulus effects were effectively due to slower response times for targets paired with the disgust-evoking pictures (see Fig. 3). Importantly, even in the first set of four test blocks (but also in all other ones), RTs were comparable for the neutral and fear-evoking pictures. The lack of an overall effect for the fear-evoking pictures can therefore not be explained by a faster habituation rate toward these stimuli.

Due to our selection criteria, the average disgust-ratings for the disgust-evoking pictures ended up to be higher than the average fear-ratings for the fear-evoking pictures (see Table 1). The main reason for this mismatch was that in our stimulus selection study, pictures with 


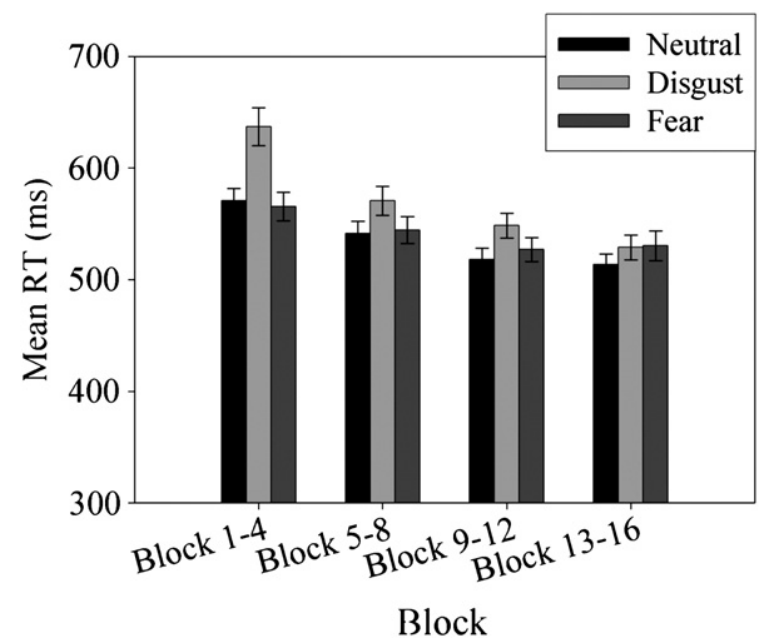

Fig. 3. Mean reaction time results for each stimulus type (neutral, disgust, fear) as a function of test quarter (blocks $1-4,5-8,9-12,13-16$ ).

higher fear-ratings also received higher disgust-ratings, creating insufficient discrimination between the emotions of interest (see also, Libkuman et al., 2007; Mikels et al., 2005). To investigate whether this difference in 'emotion strength' has contributed to the observed effects in the 200 ms cue-target interval condition, we compared participants' responses (accuracy and RT) for the five fear-evoking pictures that received the highest fear-ratings in the stimulus selection study (Mean Fear rating $=3.48$ ) with those for the five disgust-evoking pictures that received the lowest disgust-ratings (Mean Disgust rating $=3.81$ ). For accuracy, a significant effect of stimulus $(F(1,29)=4.45, p<0.05$; $\eta_{p}{ }^{2}=0.13$ ) was found, revealing poorer target identification for lowdisgust evoking pictures $(\mathrm{M}=0.78)$ compared to high-fear evoking pictures $(M=0.83)$. For RT a similar pattern emerged with slower response times for the low-disgust pictures $(\mathrm{M}=581 \mathrm{~ms})$ relative to the high-fear ones $(\mathrm{M}=545 \mathrm{~ms})\left(\mathrm{F}(1,29)=14.25, p<0.001, \eta_{p}{ }^{2}=\right.$ 0.33 ). These results endorse our main findings and suggest that the strength of the evoked emotion played no major role in obtaining our overall results. Interestingly, the distinction between high- and lowfear pictures corresponded exactly with a classification made on the basis of the pictures' biological relevance, in that the high-fear pictures were more biologically relevant (i.e., aggressive animals) than the lowfear pictures (i.e., guns and traffic accidents). In contrast, all ten disgustevoking pictures could be considered to be biologically relevant (i.e., vomit, dirt, dead animals, rotten teeth, etc.). Consequently, the above findings also rule out a potentially confounding influence of 'biological relevance'. 2

\section{Discussion}

When carefully controlling the specific emotion that is elicited by a set of negative, arousing IAPS pictures, only those evoking disgust were found to produce significant attention bias effects. More specifically, when targets were presented $200 \mathrm{~ms}$ following the onset of a central picture cue, they were identified less accurately and more slowly when these pictures were disgust-evoking than when they were fear-evoking or neutral. In addition, because similar results were not

\footnotetext{
${ }^{2}$ As a final check, an additional ANOVA was performed on the accuracy and RT results for the negative images with Emotion (fear, disgust), and Image (1-10) as within-subjects factors. For both accuracy and RT, the Emotion $\times$ Image interactions were not significant, revealing that differences between individual fear- and disgustevoking pictures were negligible and ruling out the possibility that the emotion effects were driven by a subset of images. The pictures used were thus rather homogeneous in their fear and disgust evoking qualities.
}

found for longer cue-target intervals, it seems that this bias, attributed to impaired attention disengagement by Fox et al. (2001), is rather short-lived. These results are in broad agreement with our expectations and with results from other studies that compared involuntary attention effects for disgusting and fearful pictures (Carretié et al., 2011) and for disgust- and fear-related words (Charash \& McKay, 2002; Cisler, Olantunji, et al., 2009). On the other hand, the complete absence of an effect for the fear-evoking images was not anticipated and also contrasts with findings from some earlier studies (e.g., Fox et al., 2001; Koster et al., 2004, 2006).

The prolonged RTs for targets paired with the disgust-evoking pictures might be explained by the notion that, at very short cue-target intervals (200 ms), more time and attention resources are needed to fully assess the potential risk implied in these images. In contrast, full assessment may not have been necessary for the fear-evoking images because it might have been sufficiently clear at an early stage of processing (i.e., before $200 \mathrm{~ms}$ ) that they were hazardous, allowing fast disengagement and quick responding. Indeed, such explanation would make evolutionary sense, as potentially dangerous and lifethreatening circumstances require quick action and thus a rapid shift of attention away from its source. In contrast, filthy or horrible situations may not demand an immediate action, but instead would call for a more thorough assessment of the circumstances to reveal the exact risk (e.g. contamination). Carretié et al. (2011) formulated this account in terms of costs and benefits, suggesting that less costs are associated with further exploration of disgusting pictures as compared to fearful ones. Indeed, it seems more useful if threatening stimuli are quickly detected with no additional attention so that all available resources can be directed toward finding and executing the appropriate response.

Even though this interpretation sounds plausible, the absence of RT differences between neutral and fear-evoking pictures needs further consideration, as it contrasts with several studies showing an attention bias toward threatening materials (Fox et al., 2001; Koster et al., 2004, 2006). On the other hand, it should be noted that fearrelated attention biases have been demonstrated most consistently in high-anxious participants (see reviews by Bar-Haim et al., 2007; Cisler, Bacon, et al., 2009; Yiend, 2010) and only two of our participants could be considered high-anxious relative to the rest of the sample. It is therefore likely that the fairly standard anxiety levels of our participants have contributed to the lack of significant findings for the fear-evoking images. In comparison, the response delay observed for the disgust-evoking images in the $200 \mathrm{~ms}$ interval condition occurred despite normal DS-R scores and was not modulated by the participants' level of disgust sensitivity. As already mentioned in the results section, a second reason for the fear null-effect could be that the fear-evoking pictures might have affected responses at the start of the task, but were not observed in the overall analyses because of quick habituation. We excluded this possibility however, by showing that there were no RT delays for the fear-evoking pictures in the first blocks of testing. Moreover, evidence for habituation was found for the disgust-evoking pictures, producing larger RT increases at the beginning of the task, which became gradually smaller toward the end (see Fig. 3).

As a third reason, it could be argued that we didn't find an effect of fear because our set of fear-evoking pictures evoked only minor feelings of fear. This indeed could be the case, although the same essentially holds for the disgust-evoking pictures (i.e., they elicited only minor feelings of disgust). Moreover, equating the pictures for 'emotion strength' (and thereby also for biological relevance) made no difference for the key results in the $200 \mathrm{~ms}$ interval. Selecting pictures with higher fear-ratings was not possible as they also received higher disgust ratings, causing insufficient discrimination between the emotions of interest. Moreover, the flip-side of this argument is that earlier studies, which claimed to have investigated effects of fearful pictures, have in fact studied the effects of pictures that elicited fear 
and disgust. Hence, it is possible that these added feelings of disgust explain why they found an attention bias for their "threatening" materials and we didn't.

The fact that in the $200 \mathrm{~ms}$ interval condition, attention engagement effects were found exclusively for one out of the two sets of negative pictures suggests that some basic form of emotion categorization must have taken place at early perceptual processing stages, presumably before full object recognition and conscious inference (cf., Barrett \& Bar, 2009). Indeed, in support of an early differential effect for fearful and disgusting images, recent ERP research found distinct P1 amplitudes (peak latency $96 \mathrm{~ms}$ ) for fearful and disgusting IAPS pictures, suggesting that "sophisticated visual categorization within the class of threat could occur during the initial feedforward sweep of sensory processing" (Krusemark \& Li, 2011, p. 3433). In accord with this suggestion and the current results, attention biases for disgusting stimuli are thus not necessarily restricted to later, more controlled processing stages, as has sometimes been proposed. Such suggestions on the other hand were made primarily on the basis of research with verbal material (e.g., Cisler, Olantunji, et al., 2009) or facial expressions (e.g., Santos et al., 2008), which, compared to pictures, may have created less immediate- and only secondary emotional impact.

In any case, the attention engagement effect for disgust-evoking images as observed in the current study appeared to be rather shortlived. In addition, there was no evidence for attention avoidance of these images at the longer cue-target intervals. Very brief attention effects have been observed before for "threatening" pictures, typically demonstrated in spatial cueing- and probe detection tasks by the presence of an attention effect for short exposure durations and the absence- or the reversed effect for long(er) exposure durations (e.g., Koster et al., 2005, 2006; Mogg et al., 2004). In attentional blink experiments, similar inferences have been made with respect to gradually smaller emotion effects with increasing time lags (Ciesielski et al., 2010). Together, these results (including ours) demonstrate the importance of investigating not only the magnitude but also the temporal characteristics of emotion attention allocation. Most importantly, the differential attention effect for fear- and disgust-evoking images observed in the current study implies that in emotion-attention research one should look beyond the dimensions of valence and arousal.

\section{References}

Anderson, A. K., Christoff, K., Panitz, D., De Rosa, E., \& Gabrieli, J. D. E. (2003). Neural correlates of the automatic processing of threat facial signals. The Journal of Neuroscience, 23, 5627-5633.

Bar-Haim, Y., Lamy, D., Pergamin, L., Bakermans-Kranenburg, M. J., \& Van IJzendoorn, M. H. (2007). Threat-related attentional bias in anxious and nonanxious individuals: A meta-analytic study. Psychological Bulletin, 133, 1-24.

Barrett, L. F., \& Bar, M. (2009). See it with feeling: Affective predictions during object perception. Philosophical Transactions of the Royal Society. Biological Sciences, 364, 1325-1334.

Bradley, M. M., \& Lang, P. J. (1994). Measuring emotion: The self-assessment Manikin and the semantic differential. Journal of Behavioural Therapy and Experimental Psychiatry, 25, 49-59.

Bradley, M. M., \& Lang, P. J. (1999). Affective norms for English words (ANEW): Instruction manual and affective ratings. Technical Report C -1 . The Center for Research in Psychophysiology, University of Florida.

Calder, A. J., Lawrence, A. D., \& Young, A. W. (2001). Neuropsychology of fear and loathing. Nature Reviews, 2, 352-363.

Carretié, L., Ruiz-Padial, E., López-Martín, S., \& Albert, J. (2011). Decomposing unpleasantness: Differential exogenous attention to disgusting and fearful stimuli. Biological Psychology, 86, 247-253.
Charash, M., \& McKay, D. (2002). Attention bias for disgust. Journal of Anxiety Disorders, $16,529-541$.

Charash, M., McKay, D., \& DiPaolo, N. (2006). Implicit attention bias for disgust. Anxiety Stress and Coping, 19(4), 353-364.

Ciesielski, B. G., Armstrong, T., Zald, D. H., \& Olatunji, B. O. (2010). Emotion modulation of visual attention: Categorical and temporal characteristics. PLoS One, 5(11), e13860.

Cisler, J. M., Bacon, A. K., \& Williams, N. L. (2009a). Phenomenological characteristics of attention biases towards threat: A critical review. Cognitive Therapy and Research, 33, 221-234.

Cisler, J. M., Olatunji, B. O., Lohr, J. M., \& Williams, N. L. (2009b). Attentional bias differences between fear and disgust: Implications for the role of disgust in disgustrelated anxiety disorders. Cognition \& Emotion, 23, 675-687.

Dickie, E. W., \& Armony, J. L. (2008). Amygdala responses to unattended fearful faces: Interaction between sex and trait anxiety. Psychiatry Research, 162(1), 51-57.

Fox, E., Russo, R., Bowles, R., \& Dutton, K. (2001). Do threatening stimuli draw or hold visual attention in subclinical anxiety? Journal of Experimental Psychology. General, 130, 681-700.

Koster, E. H. W., Crombez, G., Van Damme, S., Verschuere, B., \& De Houwer, J. (2004). Does imminent threat capture and hold attention? Emotion, 4, 312-317.

Koster, E. H. W., Crombez, G., Verschuere, B., Van Damme, S., \& Wiersema, J. R. (2006) Components of attentional bias to threat in high trait anxiety: Facilitated engagement, impaired disengagement, and attentional avoidance. Behaviour Research and Therapy, 44, 1757-1771.

Koster, E. H. W., Verschuere, B., Crombez, G., \& Van Damme, S. (2005). Time-course of attention for threatening pictures in high and low trait anxiety. Behaviour Research and Therapy, 43, 1087-1098.

Krusemark, E. A., \& Li, W. (2011). Do all threats work the same way? Divergent effects of fear and disgust on sensory perception and attention. The Journal of Neuroscience, 31, 3429-3434.

Lang, P. J., Bradley, M. M., \& Cuthbert, B. N. (2008). International affective picture system (IAPS): Affective ratings of pictures and instruction manual. Technical Report A-8. Gainesville, FL: University of Florida.

Libkuman, T. M., Otani, H., Kern, R., Viger, S. G., \& Novak, N. (2007). Multidimensional normative ratings for the International Affective Picture System. Behavior Research Methods, 39, 326-334.

Mikels, J. A., Fredrickson, B. L., Larkin, G. R., Lindberg, C. M., Maglio, S. J., \& ReuterLorenz, P. A. (2005). Emotional category data on images from the International Affective Picture System. Behavior Research Methods, 37, 626-630.

Mogg, K., \& Bradley, B. P. (1998). A cognitive-motivational analysis of anxiety. Behaviour Research and Therapy, 36, 809-848.

Mogg, K., Bradley, B. P., Miles, F., \& Dixon, R. (2004). Time course of attentional bias for threat scenes: Testing the vigilance-avoidance hypothesis. Cognition and Emotion, 18, 689-700.

Murphy, F. C., Nimmo-Smith, I., \& Lawrence, A. D. (2003). Functional neuroanatomy of emotions: A meta-analysis. Cognitive, Affective, \& Behavioral Neuroscience, 3, 207-233.

Olatunji, B. O., Williams, N. L., Tolin, D. F., Abramowitz, J. S., Sawchuk, C. N., Lohr, J. M. et al. (2007). The disgust scale: Item analysis, factor structure, and suggestions for refinement. Psychological Assessment, 19, 281-297.

Ploeg, H. M., van der, Defares, P. B., \& Spielberger, C. D. (1980). Handleiding bij de zelfbeoordelingsvragenlijst, ZBV. Een Nederlandstalige bewerking van de Spielberger State-Trait Anxiety Inventory. Lisse: STAI-DY. Swets en Zeitlinger.

Pratto, F., \& John, O. P. (1991). Automatic vigilance: The attention-grabbing power of negative social information. Journal of Personality and Social Psychology, 61, 380-391.

Santos, I. M., Iglesias, J., Olivares, E. I., \& Young, A. W. (2008). Differential effects of objectbased attention on evoked potentials to fearful and disgusted faces. Neuropsychologia $46,1468-1479$.

Schimmack, U. (2005). Attentional interference effects of emotional pictures: Threat, negativity, or arousal? Emotion, 5, 55-60.

Susskind, J. M., Lee, D. H., Cusi, A., Feiman, R., Grabski, W., \& Anderson, A. K. (2008). Expressing fear enhances sensory acquisition. Nature Neuroscience, 11, 843-850.

Vogt, J., De Houwer, J., Koster, E. H. W., Van Damme, S., \& Crombez, G. (2008). Allocation of spatial attention to emotional stimuli depends upon arousal and not valence. Emotion, 8, 880-885.

Vuilleumier, P. (2005). How brains beware: Neural mechanisms of emotional attention. Trends in Cognitive Sciences, 9, 585-594.

Weierich, M. R., Treat, T. A., \& Hollingworth, A. (2008). Theories and measurement of visual attentional processing in anxiety. Cognition \&' Emotion, 22, 985-1018.

Weinberg, A., \& Hajcak, G. (2010). Beyond good and evil: The time-course of neural activity elicited by specific picture content. Emotion, 10, 767-782.

Yiend, J. (2010). The effects of emotion on attention: A review of attentional processing of emotional information. Cognition E' Emotion, 24, 3-47. 